\title{
Generation of Robust Hyperbolic Chaos in CNN
}

\section{S. P. Kuznetsov}

It is shown that on the basis of a cellular neural network (CNN) composed, e.g., of six cells, it is possible to design a chaos generator with an attractor being a kind of Smale-Williams solenoid, which provides chaotic dynamics that is rough (structurally stable), as follows from respective fundamental mathematical theory. In the context of the technical device, it implies insensitivity to small variations of parameters, manufacturing imperfections, interferences, etc. Results of numerical simulations and circuit simulation in the MULTisim environment are presented. The proposed circuit is the first example of an electronic system where the role of the angular coordinate for the Smale-Williams attractor is played by the spatial phase of the sequence of patterns. It contributes to the collection of feasible systems with hyperbolic attractors and thus promotes filling with real content and promises practical application for the hyperbolic theory, which is an important and deep sector of the modern mathematical theory of dynamical systems.

Keywords: cellular neural network, chaos, attractor, pattern, dynamics, structural stability

\section{Introduction}

Cellular neural networks (CNN) are defined as arrays composed of cells arranged in space, each of which is governed by a set of ordinary nonlinear differential equations and interacts with the neighboring cells [1-3]. The paradigm of CNN based on electronic components was proposed about thirty years ago. Such devices can be used as analog processors capable of highly efficient parallel data processing, as an alternative to traditional computational approaches. There is an extensive literature on various aspects of the theoretical analysis of CNN, their circuit design, including that at the level of microchips, as well as on their use for image processing, system control in robotics, neural system modeling and so on [4-10].

Received April 15, 2019

Accepted May 02, 2019

The work was carried out within the framework of the state task of Kotel'nikov's IRE RAS.

Sergey P. Kuznetsov

spkuz@yandex.ru

Kotel'nikov Institute of Radio Engineering and Electronics, Russian Academy of Sciences, Saratov Branch ul. Zelenaya 38, Saratov, 410019 Russia 
Of interest may be the use of cellular neural networks to construct generators of robust chaos.

Dynamic chaos is believed to have great potential for a variety of practical applications, such as secure communication [11-13], data encryption [15-17], pseudo-random number generation [18-21], development of novel effective radar and sonar technologies [22-26]. For such applications, it is essential for the chaotic dynamics to persist when the system parameters are varied, i.e., the property of robustness takes place. Robust chaos [25-29] contrasts with the "fragile chaos" most often encountered in dynamical systems and associated with quasi-attractors [30]. In the latter case, the dynamics observed in numerical calculations or in experiments, although it looks chaotic, is also characterized by the presence of regular orbits with narrow basins of attraction in the nearby region of the phase space, or they appear at small parameter variations.

To date, there has been a rather extensive literature devoted especially to the problem of generation of robust chaos. For this purpose, various approaches have been proposed, for example, the use of systems reproducing the dynamics of one-dimensional mappings, piecewise smooth two-dimensional mappings, such as Lozi [31,32] and Belykh [33, 34] maps, or the dynamics on Lorentz-type attractors. (See a review of the approaches in [27, 28].)

However, in our opinion, the most fundamental approach is using systems that demonstrate rough hyperbolic chaos, which makes it possible to rely on the results of great advances in mathematical theory in the $1960 \mathrm{~s}-70$ s, sometimes referred to as the "hyperbolic revolution" [35-38]. The hyperbolic attractors introduced into consideration in the framework of this theory are characterized by a rigorously substantiated property of roughness or structural stability [39], which consists in the fact that, with a small variation of parameters or functions involved in the equations, the chaotic dynamics remain essentially the same, up to a continuous change of variables. From the point of view of physical or technical objects, this means insensitivity of the generated dynamics to small variations in the system component characteristics, manufacturing imperfections, interferences, aging, etc.

It should be noted that the design of systems with hyperbolic attractors requires special efforts, and until recently, well-known examples were limited to mathematical constructions. One of such examples is the Smale-Williams solenoid [30, 36-38]. Suppose that, in the phase space of some abstract dynamical system, a torus-shaped domain filled with points representing instantaneous states undergoes in one step of evolution a stretch in the longitudinal direction by an integer number of times $M$ together with strong transversal compression and folding into a loop placed inside the original torus, as shown in Fig. 1. (This illustration refers to the special case $M=2$.) At each step of iteration, the volume of the object decreases, and the number of turns grows by factor $M$. In the limit, an object arises with an infinite number of turns, which has a Cantor structure in the transversal cross-section that is the Smale-Williams attractor. The essential point is that one of the dynamical variables, the angular coordinate $\varphi$, undergoes in one step a transformation of such topological nature that one round of the full circle for the preimage corresponds to an $M$-fold round for the image (no matter whether in forward or backward direction). The dynamics of an individual representative point in accordance with this mapping is chaotic. Although the described construction appeals for clarity to the threedimensional state space, this point is not significant, and attractors of this type can also take place in state spaces of higher dimensions.

Recently, approaches to construction of feasible systems with hyperbolic attractors have been proposed, and chaos generated by them has been demonstrated in computations [40, 41], as well as in several experiments with laboratory electronic devices [45-48]. 


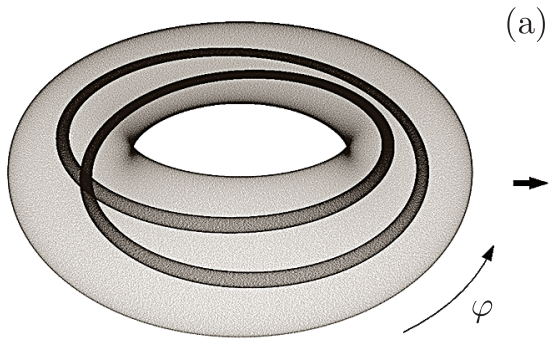

(a)
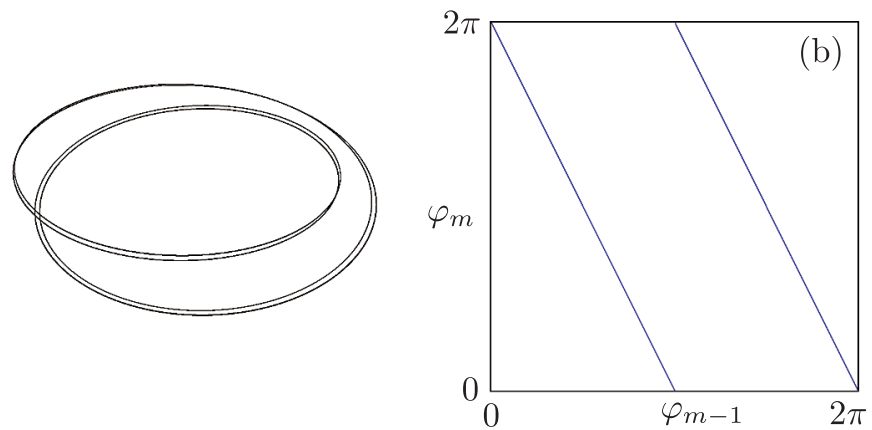

Fig. 1. (a) Transformation of a torus region in one evolutionary step in time and a limit object, the attractor in the form of a solenoid, depicted for the case of a ratio of stretching along the angular coordinate $M=2$. (b) A plot of the angular variable at discrete time $m$ as a function of the previous value. In this case, a round of the complete circle for preimage corresponds to a double round for the image in the opposite direction.

Returning to the CNN problems, it should be noted that one of the actively developed areas within this paradigm is to use them for analog modeling of complex space-time dynamics including such phenomena as formation of Turing structures, autowaves, spiral patterns, and turbulent behavior [8-10].

In [49], for a one-dimensional medium capable of generating Turing patterns, the idea of organizing a Smale-Williams attractor with $M=3$ was proposed. It corresponds to a situation where due to periodic modulation of a parameter, long-wave and short-wave structures alternate, and the spatial phase of the patterns undergoes successive transformation according to the expanding circle map. A solenoid appears here as an object embedded in an infinite-dimensional state space of the mapping describing the evolution of states of the distributed system over a modulation period. In [50] it was shown that it is possible to implement a similar mechanism of formation of the Smale-Williams attractor at parametric excitation of standing waves in a ring nonlinear system, when the pumping is alternately provided at a frequency corresponding to the main parametric resonance and at the tripled frequency.

In [51] an example of an autonomous distributed ring system is given, where the attractor occurs in the form of a Smale-Williams solenoid with the index $M=2$ for the mapping describing a state transformation over a characteristic time period of onset and suppression of the activity.

In view of the above results, a natural idea comes up of using CNN to construct rough hyperbolic chaos generators. This article demonstrates the possibility of implementing the SmaleWilliams attractor in an electronic cellular neural network reproducing the space-time dynamics similar to that discussed in [51].

Section 2 formulates the basic equations of the system that is a chain of cells with a certain type of dynamics, and explains the mechanism for the appearance of the hyperbolic chaos due to the expanding map for the spatial phases of the patterns formed at successive stages of activity of the cells. Section 3 presents and discusses results of numerical simulation of the dynamics based on numerical integration of the proposed equations. Section 4 discusses the CNN designed as an electronic device that implements chaotic dynamics on the Smale-Williams attractor. Also, results of circuit simulating for operation of the device in the MULTisim environment are presented and compared with the results of numerical integration of the equations. The Conclusion discusses the significance of the results obtained and prospects for further research. 


\section{Main equations and mechanism of operation}

Consider a system that is a ring chain of $2 N$ cells governed by differential equations of the following form:

$$
\begin{aligned}
& \dot{u}_{n}=D\left(u_{n-1}-2 u_{n}+u_{n+1}\right)+u_{n}^{3}-u_{n} v_{n}^{2}-\alpha u_{n+N}+\varepsilon \delta_{n} v_{n} \\
& \dot{v}_{n}=\left(-\gamma+u_{n}^{2}\right) v_{n}+\mu u_{n}^{2} .
\end{aligned}
$$

Here $u_{n}, v_{n}$ are the dynamical variables that determine instantaneous states of the cells numbered by $n=0,1, \ldots 2 N-1$, and $\mu, \varepsilon, \gamma$ are parameters. In addition to the coupling of neighboring cells, characterized by the coefficient $D$, it is assumed that there are still links between each cell and the opposite element of the ring structure, characterized by parameter $\alpha$. By means of a set of values $\delta_{n}$, a weak constant in time spatial inhomogeneity is introduced, the role of which will be explained below.

If we neglect the spatially inhomogeneous additive (i.e., we set $\varepsilon=0$ ), then, near the trivial equilibrium state of the system (2.1) $u_{n}=0, v_{n}=0$, the substitution $u_{n} \sim \exp \left(\lambda t-i \pi k n N^{-1}\right)$ leads to the following expression for the increments of modes characterized by wavenumbers $k$, which, in accordance with the boundary conditions of periodicity, should be taken as integers:

$$
\lambda(k)=-\alpha(-1)^{k}-4 D \sin ^{2}(\pi k / 2 N) .
$$

As seen from the formula (2.2), under the condition $0<\alpha<4 D \sin ^{2}(3 \pi / 2 N)$, only one mode $k=1$ has a positive increment and is unstable, while the other modes are damped, including the homogeneous one with $k=0$.

When specifying the spatial inhomogeneity in such a form that the third spatial harmonic yields the main contribution, i.e., $\delta_{n} \sim \cos 3 \pi n N^{-1}+\ldots$, it is possible to ensure the functioning of the system in a regime of hyperbolic chaos, which is qualitatively described as follows.

First, let the system be close initially to the trivial state $u_{n}=0, v_{n}=0$ and demonstrate a growth in time of the spatial distribution $u_{n}$ with the wave number $k=1$. In the general case, this is a superposition of the sine and cosine components with some coefficients that can be written as a single term characterized by some spatial phase $\varphi: u_{n} \sim \cos \left(\pi n N^{-1}+\varphi\right)$.

When the factor $\left(-\gamma+u_{n}^{2}\right)$ in the second equation (2.1) becomes positive, the growth of the variable $v_{n}$ begins. Since at its initiation the process is stimulated by a quadratic term $u_{n}^{2}$, the spatial dependence of $v_{n}$ will be determined by the second harmonic: $v_{n} \sim \cos ^{2}\left(\pi n N^{-1}+\right.$ $+\varphi)=\frac{1}{2}+\frac{1}{2} \cos \left(2 \pi n N^{-1}+2 \varphi\right)$. As the values of $v_{n}$ grow, the variables $u_{n}$ start to decrease rapidly from a certain time due to the inhibitory effect of the term in the first equation (2.3) proportional to $v_{n}^{2}$. When the values of $u_{n}$ become small enough, the variables $v_{n}$ also experience damping with decrement determined by the parameter $\gamma$.

Further, there comes a new stage of increase of $u_{n}$, stimulated at its initial part by a term $\varepsilon \delta_{n} v_{n}$ in the first equation, namely, by its first spatial harmonic. The first harmonic appears as a result of combination of the second harmonic of the variable $v_{n}$ and the third harmonic of the function describing spatial inhomogeneity: $\delta_{n} v_{n} \sim \cos 3 \pi n N^{-1} \cos \left(2 \pi n N^{-1}+2 \varphi\right)=$ $=\frac{1}{2} \cos \left(\pi n N^{-1}-2 \varphi\right)+\ldots$ This ensures the transfer of the double phase with the opposite sign to the first harmonic of the variable $u_{n}: \varphi_{m+1} \approx-2 \varphi_{m}$. Thus, for the spatial phase, an expanding circle map takes place, which produces chaotic dynamics with Lyapunov exponent $\Lambda \approx \ln 2=0.693 \ldots$

Similar dynamics can be provided in CNN composed of twice less number of cells. Indeed, taking into account the fact that the spatial structure of the periodic continuation of $u_{n}$ is 
determined by odd harmonics, and for $v_{n}$ by even ones, the class of solutions of (2.1) we consider must satisfy the conditions $u_{n+N}=-u_{n}$ and $v_{n+N}=v_{n}$. Therefore, instead of (2.1) we can write the system of equations for $N$ cells

$$
\begin{aligned}
& \dot{u}_{n}=D\left(u_{n-1}-2 u_{n}+u_{n+1}\right)+u_{n}^{3}-u_{n} v_{n}^{2}+\alpha u_{n}+\varepsilon \delta_{n} v_{n} \\
& \dot{v}_{n}=\left(-\gamma+u_{n}^{2}\right) v_{n}+\mu u_{n}^{2}
\end{aligned}
$$

assuming that the boundary conditions of periodicity for $u_{n}$ are replaced by the conditions of a sign change at the ends:

$$
u_{-1}=-u_{N-1}, u_{N}=-u_{0} .
$$

Note that in the system (2.3), unlike (2.1), long-distance interactions of cells with opposite elements are excluded; the corresponding term in the equation contains a variable related to the same cell, with the inverted sign.

The state space of the system (2.3) is of dimension $2 N$. The system can also be described in terms of the Poincaré map, the dimension of which is $2 N-1$. To do so, we need to introduce a cross-section in the state space of the system (2.3) by some hypersurface $S$, which is given by some algebraic equation $f\left(u_{0}, v_{0}, \ldots, u_{N-1}, v_{N-1}\right)=0$ and must cross the flow of phase trajectories. The Poincaré map expresses the vector of a point on the hypersurface through the vector of the previous point of its intersection by the trajectory: $\mathbf{X}_{m}=\mathbf{F}\left(\mathbf{X}_{m-1}\right)$. The Poincaré map can be implemented as a computer program that performs integration of the system of differential equations by a finite-difference method from one to another intersection of the hypersurface $S$, taking the vector $\mathbf{X}_{m-1}$ as the initial condition and having the vector $\mathbf{X}_{m}$ as the result.

When the system operates according to the mechanism described above, the Smale-Williams type attractor has to take place in the state space of the Poincaré map, and the spatial phase of the patterns appearing at successive stages of activity plays the role of the angular variable for the solenoid.

\section{Numerical results}

Figure 2 shows space-time diagrams illustrating the dynamics of the ring system with the number of cells $2 N=12$, obtained by numerical integration of Eqs. (2.1) by the fourth-order Runge - Kutta method. The distributions of the values $u$ and $v$ are shown depending on the spatial index $n$ plotted along the horizontal axis at time instants corresponding to maximal values of the first mode amplitude estimated as $\sqrt{u_{0}^{2}+u_{N / 2}^{2}}$. Although $u_{n}$ and $v_{n}$ variables relate to discrete spatial points, the corresponding dots in the diagram are linked by straight line segments to visualize clearly the structure of the spatial patterns. The calculations were carried out with the values of parameters

$$
D=8, \alpha=2.2, \gamma=0.6, \mu=0.4, \varepsilon=0.25,
$$

adding the spatial inhomogeneity corresponding to the third spatial harmonic with the set of values $\delta_{n}=\{1,-1,-1,1,1,-1,-1,1,1,-1,-1,1\}$. As seen from the figure, the waveforms at each new stage of activity jump randomly over the chain length.

Similar dynamics is observed for the same parameters in the numerical simulations of the system (2.3) with the number of cells $N=6$ and the boundary conditions of sign reversal at the ends (2.4), and with the inhomogeneity given by the set $\delta_{n}=\{1,-1,-1,1,1,-1\}$. 

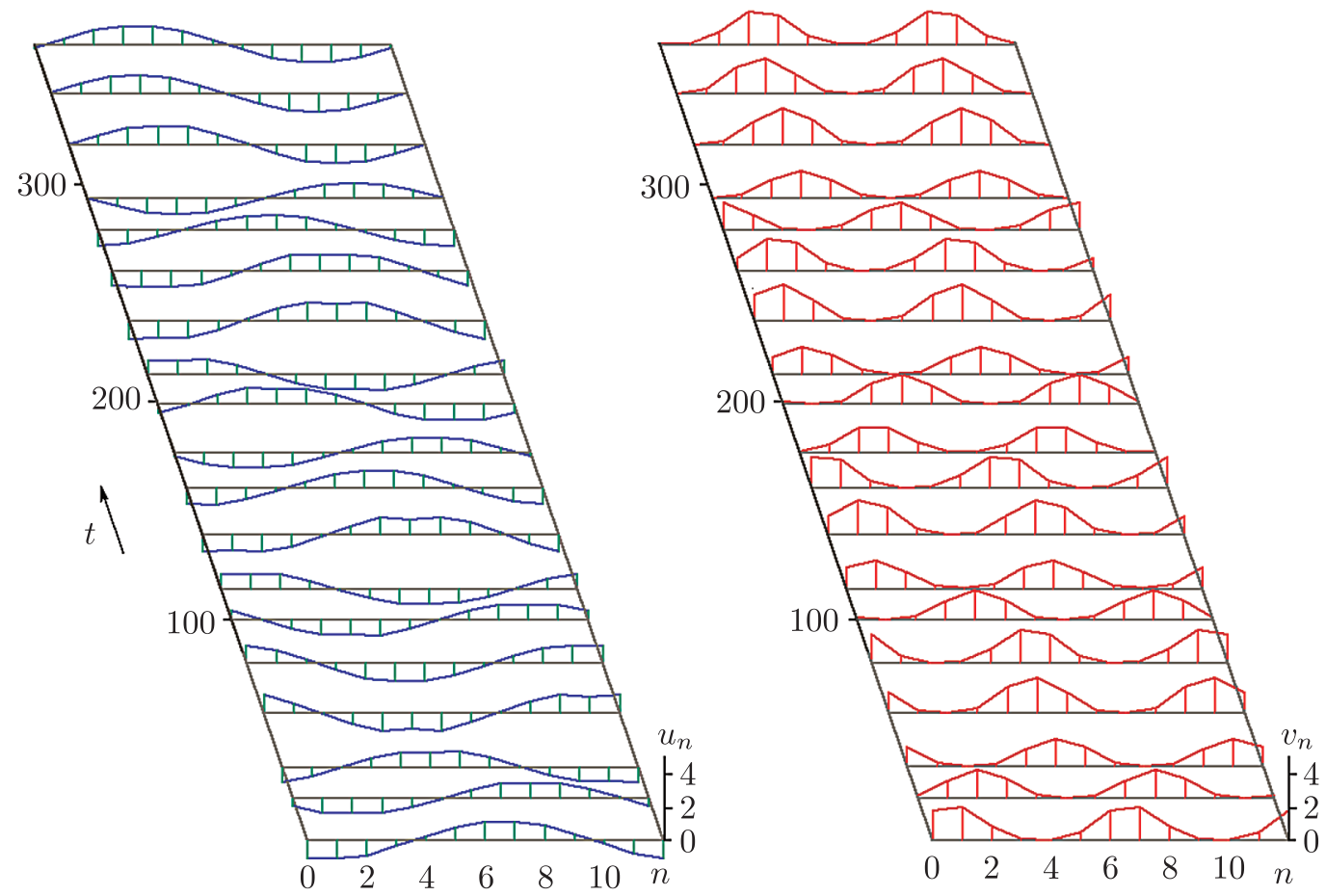

Fig. 2. Evolution of patterns in the ring system (2.1) with the number of cells $2 N=12$ in the sustained chaotic regime, on the left for the variable $u$, and on the right for the variable $v$. The configurations relating to the moments of successive maximums of the value $\sqrt{u_{0}^{2}+u_{3}^{2}}$ are shown. The parameters are: $D=8, \alpha=2.2, \gamma=0.6, \mu=0.4, \varepsilon=0.25, \delta_{n}=\{1,-1,-1,1,1,-1,-1,1,1,-1,-1,1\}$.

It can be verified that the observed chaotic displacement of the patterns at successive stages of activity corresponds to the transformation of the spatial phase according to the double expanding circle map. For this, in the process of numerical integration of the equations, at an instant of each $m$ th maximum of the first mode amplitude $\sqrt{u_{0}^{2}+u_{N / 2}^{2}}$, the spatial phase of the pattern is evaluated as $\varphi_{m}=\arg \left(u_{0}+i u_{N / 2}\right)$, and the data are plotted in coordinates $\left(\varphi_{m-1}, \varphi_{m}\right)$.

Figure 3 combines the graphs obtained by processing the numerical integration data for the model (2.1) (red points) and for the model (2.3) (blue points). It can be seen that the points fit the same lines, characterized by an average slope -2 .

Although the shape of the branches in Fig. 3 is distorted in comparison with the ideal linear function of Fig. 1b, this does not violate affiliation of the map to the same topological class. Indeed, a single passage of a full phase interval along the abscissa axis for the preimage corresponds to a double passage for the image along the ordinate axis (in the opposite direction). This circumstance, in the presence of a sufficient degree of compression of the phase volume in other directions in the state space ensures the existence of the attractor of Smale-Williams type.

Figure 4 illustrates the dependence of the dynamical variables belonging to a single cell of the system on time in the regime of sustained chaotic self-oscillations as obtained from numerical integration of Eqs. (2.3). Figure 5 for the same case shows portraits of the attractor in different representations. Panel (a) shows a projection of the trajectory belonging to the attractor onto the phase plane of the individual cell $\left(u_{0}, v_{0}\right)$. Panel (b) represents the attractor in projection 


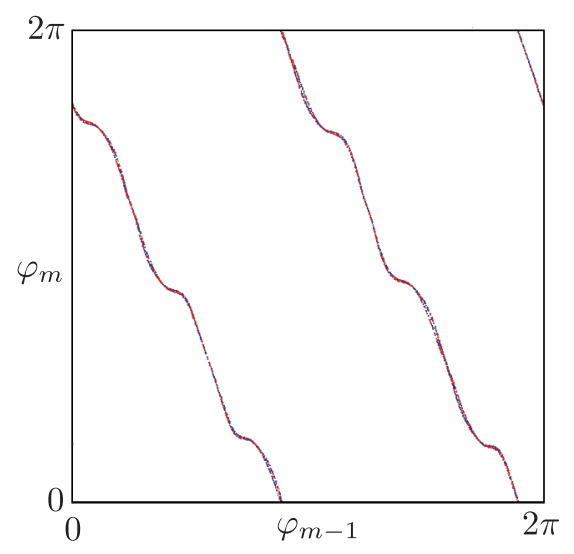

Fig. 3. Diagram for spatial phases calculated as $\varphi_{m}=\arg \left(u_{0}+i u_{N / 2}\right)$ on reaching the $m$ th maximum of the value $\sqrt{u_{0}^{2}+u_{N / 2}^{2}}$. Red dots refer to the system (2.1) of $2 N=12$ cells, and the blue dots belong to the system (2.3) of $N=6$. The diagram should be compared with Fig. 1c.

on the plane of variables related to cells separated by a quarter wavelength of the main mode $\left(u_{0}, u_{N / 2}\right)$. These pictures illustrate the chaotic nature of the dynamics and will be useful for comparison with the data of the circuit simulation in the next section, but it is difficult to judge from them about the nature of the attractor in the sense of its relation to the Smale-Williams solenoid. To make this connection visible, Fig. $4 \mathrm{c}$ shows the set of points $\left(u_{0}, u_{N / 2}\right)$ corresponding to the instants when the amplitude of the first mode evaluated as $\sqrt{u_{0}^{2}+u_{N / 2}^{2}}$ reaches maximal values. This image can be interpreted as a portrait of the attractor in a Poincaré section, in a two-dimensional projection, similar to the Smale-Williams solenoid depicted in Fig. 1a. An enlarged fragment in the center of the plot in Fig. 4c illustrates the transversal Cantor structure inherent in the solenoid.
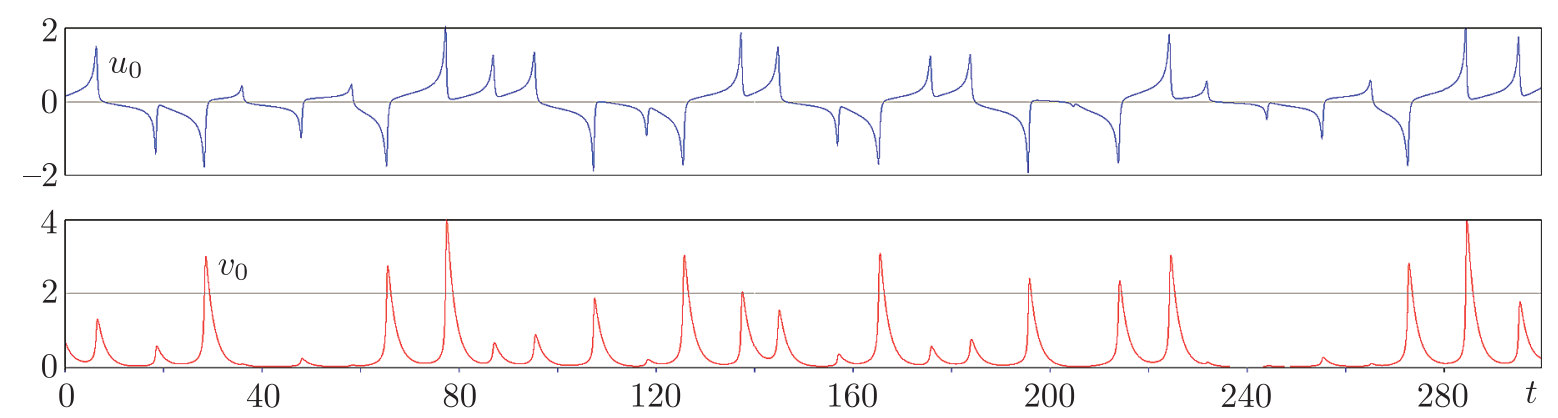

Fig. 4. Time dependence of the dynamical variables $u$ and $v$ in the cell $n=0$ in the sustained regime of chaotic self-oscillations as obtained from the numerical integration of Eqs. (2.3). The parameters are: $D=8, \alpha=2.2, \gamma=0.6, \mu=0.4, \varepsilon=0.25, \delta_{n}=\{1,-1,-1,1,1,-1\}$.

To characterize the chaotic dynamics on the attractor quantitatively, it is natural to turn to calculation of the Lyapunov exponents. The total number of Lyapunov exponents for the system (2.3) is $2 N=12$, however, in order to judge on the chaotic nature of the attractor and its fractal properties, it is sufficient to evaluate only several of the largest exponents. Calculations are made according to the standard algorithm [44, 52-54], which includes the joint solution of Eqs. (2.3) and a set of variation equations for small perturbations in a number equal to the 

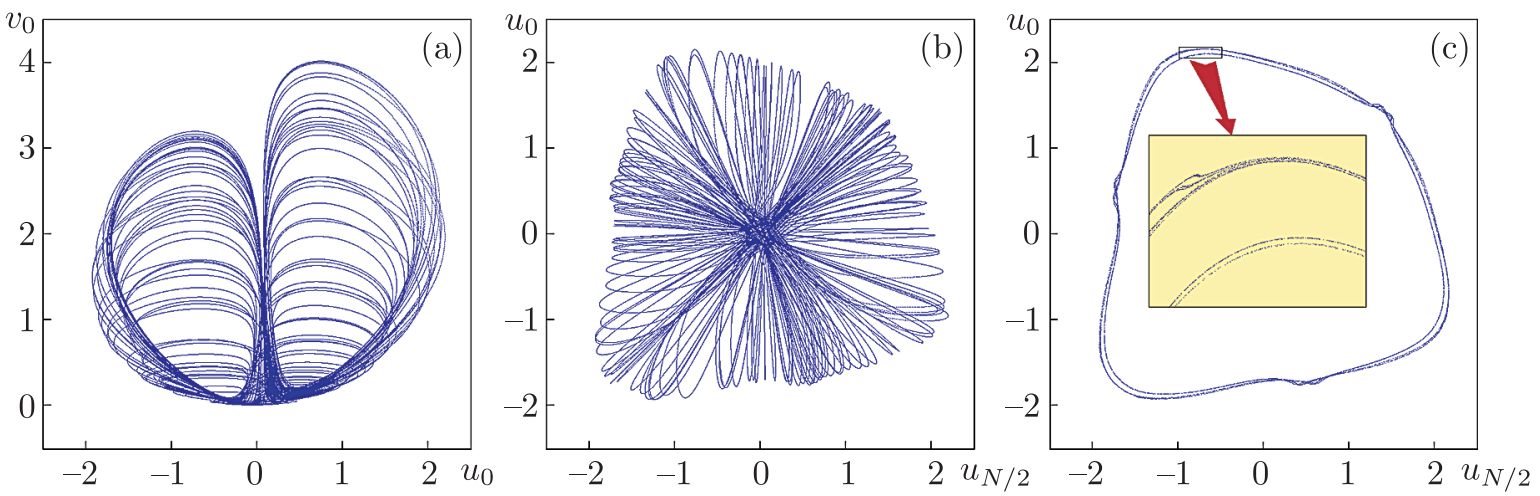

Fig. 5. Portraits of a trajectory belonging to the attractor of the system (2.3) in projection on the phase plane of the individual cell $\left(u_{0}, v_{0}\right)$ (a) and on the plane of variables related to cells separated by a quarter wavelength of the main mode $\left(u_{0}, u_{N / 2}\right)(\mathrm{b})$. The diagram on the right $(\mathrm{c})$ shows the set of

points $\left(u_{0}, u_{N / 2}\right)$ corresponding to instants of reaching maximums of the value $\sqrt{u_{0}^{2}+u_{N / 2}^{2}}$ visualizing the Smale-Williams solenoid in the two-dimensional projection.

number of the evaluated exponents, with the Gram - Schmidt orthogonalization of the monitored perturbation vectors at each integration step. For the attractor, to which the diagrams in Fig. 5 belong, the first three exponents are

$$
\lambda_{1}=0.0597, \lambda_{2}=0.0000, \lambda_{3}=-0.2046 .
$$

With the selected parameters, the average period for successive passages of the Poincaré section according to the results of the calculations is $T \approx 9.866$, hence, for the largest Lyapunov exponent of the Poincaré map we get $\Lambda_{1}=\lambda_{1} T \approx 0.589$, which roughly agrees with the value $\ln 2=0.693 \ldots$ for the linearly expanding map of Fig. 1c. The second exponent is zero, up to the computational accuracy, and should be interpreted as that associated with a shift-type perturbation vector along the reference phase trajectory. The remaining exponents are negative. The Kaplan - Yorke dimension $[44,55,56]$ for the Poincaré map attractor is $D_{K Y}=1+\lambda_{1} /\left|\lambda_{3}\right| \approx$ 1.29 , which reflects the fractal transversal structure of the solenoid.

\section{Circuit implementation and simulations}

Figure 6 shows a circuit diagram of CNN, the dynamics of which corresponds to the model (2.3). The system is built of blocks; the circuit diagram of a single cell is shown in Fig. 7. The connection between the cells is provided by resistors $R_{1}-R_{6}$ having equal resistances $R$, and the connection of the first and the last elements of the chain is arranged in a special way to ensure the condition of sign reversal of type (2.4), while the resistors $R_{7}, R_{8}$ have the twice less resistances $R / 2$.

Consider the circuit diagram of an individual cell and derive the respective equations for it.

Note that in the working area of voltages below $\sim 0.4 \mathrm{~V}$ the source-drain conductance of the field-effect transistor $2 \mathrm{~N} 5020$, which is controlled by the source-gate voltage, may be approximated with sufficient accuracy by the expression

$$
g\left(U_{G S}\right)=g_{0}-g_{1} U_{G S},
$$

where $g_{0} \approx 0.0017 \mathrm{~A} / \mathrm{V}, g_{1} \approx 0.0020 \mathrm{~A} / \mathrm{V}^{2}$. 


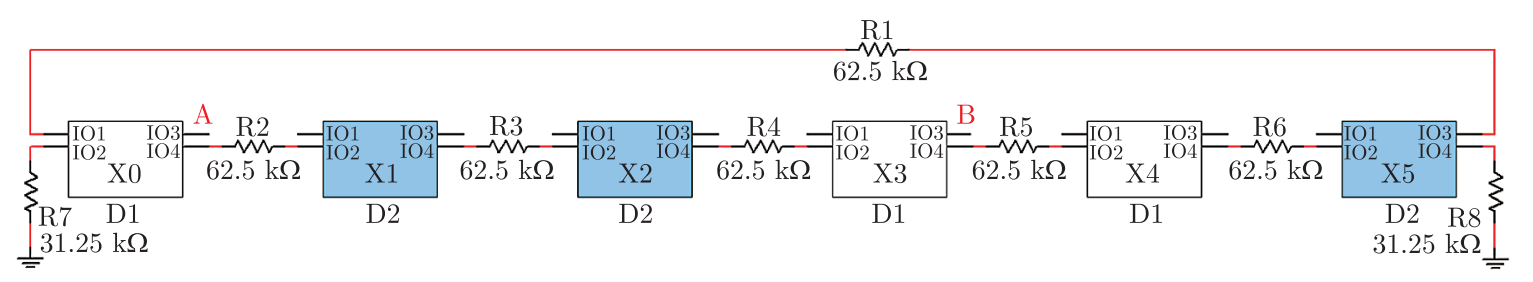

Fig. 6. Circuit diagram for the CNN, where blocks D1 and D2 differ only by the positions of the switches in the circuit of Fig. 7 .

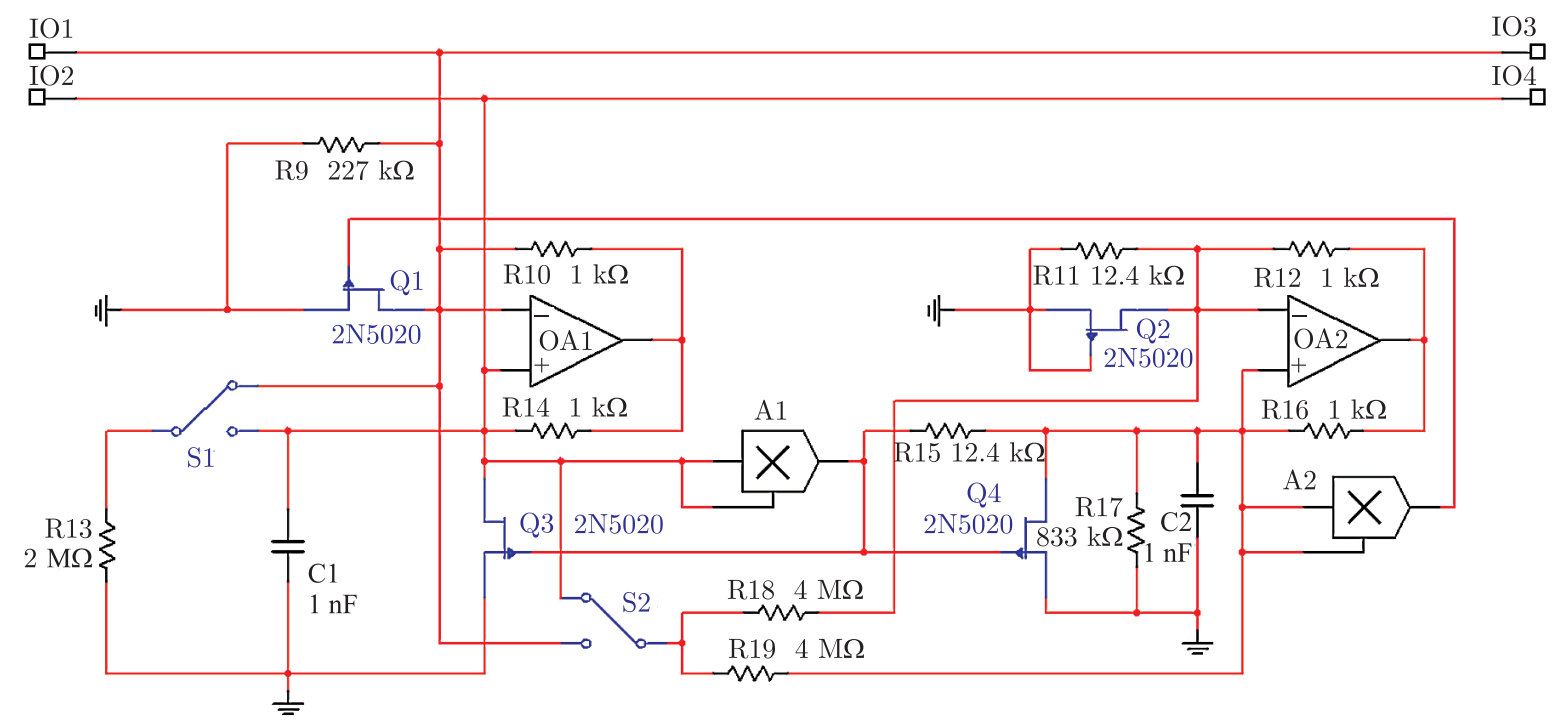

Fig. 7. Circuit diagram of an individual cell of the system, where the indicated positions of the switches $\mathrm{S} 1$ and $\mathrm{S} 2$ correspond to the block D1, and their opposite positions to the block D2.

If the instantaneous values of the voltages across the capacitors $\mathrm{C} 1$ and $\mathrm{C} 2$ are $U$ and $V$, then at the output of the analog multipliers A1 and A2, which have a transmission coefficient $0.1 \mathrm{~V}^{-1}$, the voltages are $\frac{1}{10} U^{2}$ and $\frac{1}{10} V^{2}$.

We first turn to the block D1, which corresponds to the positions of the switches shown in Fig. 7, and write down conditions for zero total current at the inverting and non-inverting inputs of the operational amplifier OA1:

$$
\begin{aligned}
& \left(R_{13}^{-1}+R_{9}^{-1}\right) U+\left(g_{0}-\frac{1}{10} g_{1} V^{2}\right) U=I+\left(W_{1}-U\right) R_{10}, \\
& C_{1} \dot{U}+\left(g_{0}-\frac{1}{10} g_{1} U^{2}\right) U+(U-V)\left(R_{18}^{-1}+R_{19}^{-1}\right)= \\
& =J+\left(W_{1}-U\right) R_{14},
\end{aligned}
$$

where $I$ is the current flowing through the terminals IO1 and IO3, and $J$ is the current flowing through the terminals IO2 and IO4, $W_{1}$ is the voltage at the output of the operational amplifier. Similar current balance equations at inputs of OA2 are

$$
\begin{aligned}
& R_{11}^{-1} V+g_{0} V=R_{18}^{-1}(U-V)+\left(W_{2}-V\right) R_{12}, \\
& C_{2} \dot{V}+R_{17}^{-1} V+\left(g_{0}-\frac{1}{10} g_{1} U^{2}\right) V+R_{15}^{-1}\left(V-\frac{1}{10} U^{2}\right)= \\
& =R_{19}^{-1}(U-V)+\left(W_{2}-V\right) R_{16} .
\end{aligned}
$$


Setting $R_{10}=R_{11}=R_{12}=R_{16}, \frac{1}{2} R_{18}=\frac{1}{2} R_{19}=R_{13}, R_{11}=R_{15}, C_{1}=C_{2}=C$, we get

$$
\begin{aligned}
& R_{0} C \dot{U}=U^{3}-U V^{2}+R_{0} R_{13}^{-1} V+R_{0} R_{9}^{-1} U+R_{0}(J-I), \\
& R_{0} C \dot{V}=-R_{0} R_{17}^{-1} V+U^{2} V+\frac{1}{10} R_{0} R_{15}^{-1} U^{2},
\end{aligned}
$$

where, for convenience, we have introduced a normalization factor with the dimension of resistance $R_{0}=5 \mathrm{k} \Omega$ so that $\frac{1}{10} g_{1} R_{0}=1$.

At the opposite positions of the switches, the equations for the D2 block are derived in a similar way, which differ from (4.4) only by the sign of the term $R_{0} R_{13}^{-1} V$ in the first equation.

When connecting the blocks to the chain, for the difference of currents one has to use the expression

$$
I_{n}-J_{n}=R^{-1}\left(U_{n-1}-2 U_{n}+U_{n+1}\right),
$$

where $n$ is the spatial index of the cells, to which the currents and voltages relate. Given the special connection at the edges of the chain, the same relationship holds true for end cells if we assume $U_{-1}=-U_{N-1}, U_{N}=-U_{0}$.

To ensure compliance with the equations of Section 2, the resistances must be assigned in such a way that the following relations are satisfied:

$$
\begin{gathered}
R_{0} R^{-1}=D, R_{0} R_{9}^{-1}=\frac{1}{100} \alpha, R_{0} R_{17}^{-1}=\frac{1}{100} \gamma \\
R_{0} R_{13}^{-1}=\frac{1}{100} \varepsilon, \quad R_{0} R_{15}^{-1}=\mu .
\end{gathered}
$$

The resulting equations are equivalent to the system (2.3)

$$
\begin{aligned}
R_{0} C \dot{U}_{n}= & U_{n}^{3}-U_{n} V_{n}^{2}+ \\
& +\frac{1}{100}\left[D\left(U_{n-1}-2 U_{n}+U_{n+1}\right)+\alpha U_{n}+\varepsilon \delta_{n} V_{n}\right], \\
R_{0} C \dot{V}_{n}= & -\frac{1}{100} \gamma V_{n}+\frac{1}{10} \mu U_{n}^{2}+U_{n}^{2} V_{n},
\end{aligned}
$$

where $\delta_{n}=\{1,-1,-1,1,1,-1\}$. The only difference is in the normalization of the variables. Namely, in order for compliance to take place, it is necessary to set the unit voltage to be $0.1 \mathrm{~V}$ and the unit time to be $100 R_{0} C=0.5 \mathrm{~ms}$. The nominal values of the components indicated in the circuit diagrams of Figs. 6 and 7 were selected to correspond to the parameters used in Section 2 .

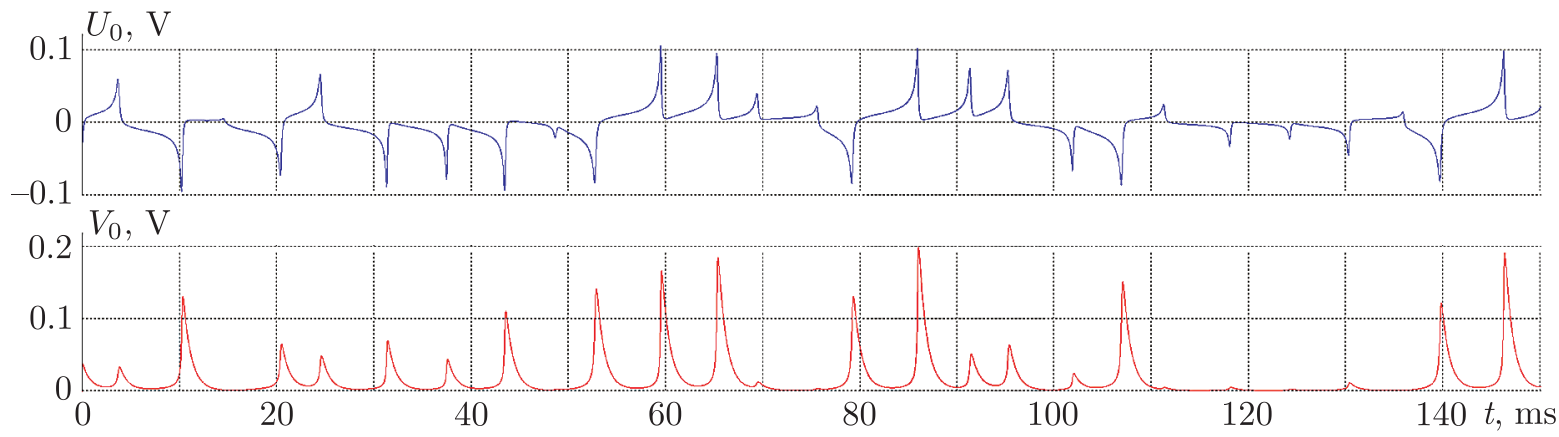

Fig. 8. Oscilloscope traces for the voltages across capacitors C1 and C2 for one of the cells D1 of the system, copied from the screen of the virtual oscilloscope in the course of simulating the circuit functioning in Multisim. 
Figure 8 shows the oscilloscope traces of voltages across the capacitors $\mathrm{C} 1$ and $\mathrm{C} 2$ for one of the cells of the system, obtained with a virtual oscilloscope in the course of simulating the circuit functioning with the MulTisim environment. ${ }^{1}$ The graphs are given in a scale allowing a direct visual comparison of them with the results of the numerical solution of the equations in Fig. 4.

Figure 9 shows the power spectrum of the signal corresponding to the voltage on the capacitor C1 of a block D1, obtained by simulating in Multisim using a virtual spectrum analyzer. The spectrum is shown in logarithmic scale. The continuous power spectrum indicates the chaotic nature of the dynamics on the attractor.

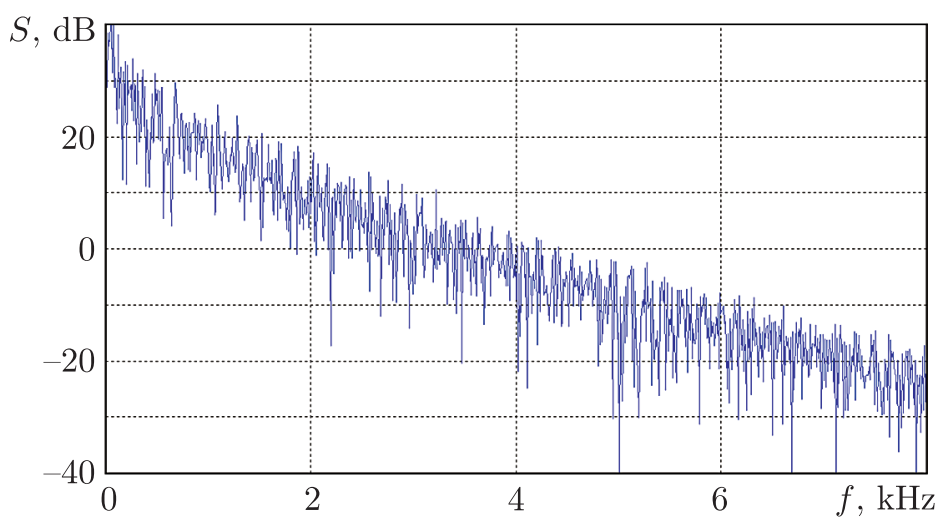

Fig. 9. Power spectrum of the signal corresponding to the voltage across the capacitor C1 of the cell $n=0$ obtained in the MULTisim simulation using a virtual spectrum analyzer.
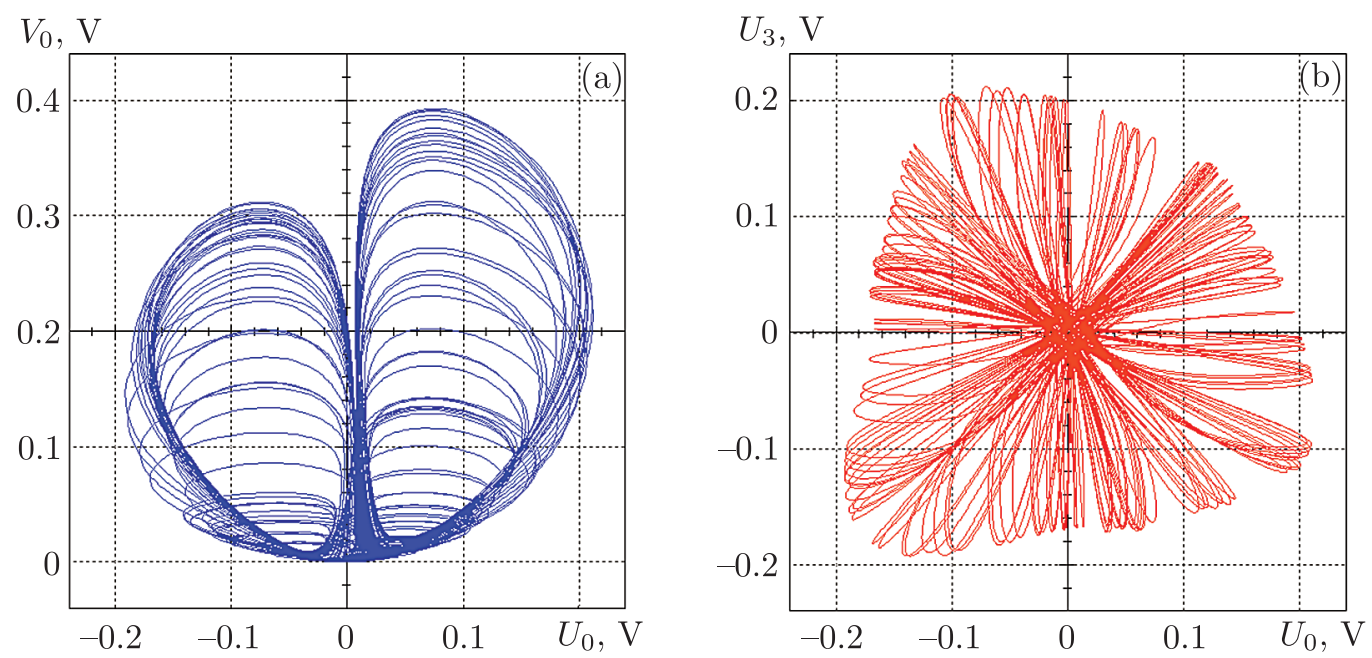

Fig. 10. Oscilloscope traces obtained in the MuLTISIM simulation corresponding to phase trajectories in projection on a plane, where the coordinates are the voltages on the capacitors $\mathrm{C} 1$ and $\mathrm{C} 2$ of a block D1 (a) and on the plane, where the coordinates are the voltages on the capacitors C1 in the cells separated by a quarter wavelength of the main mode (b).

\footnotetext{
${ }^{1}$ To ensure the departure from the trivial state $U_{n}=0, V_{n}=0$ when modeling in MuLTiSIM, it is sufficient to set an arbitrary nonzero initial voltage on one of the capacitors and in the menu item "Interactive Simulation Settings" to apply the "User defined" option for the initial conditions.
} 
Figure 10 shows portraits of the attractor obtained by simulating the circuit in MULTISIM environment. Panel (a) corresponds to the phase portrait observed when the voltages on the capacitors $\mathrm{C} 1$ and $\mathrm{C} 2$ in one of the D1 blocks are supplied to the horizontal and vertical inputs of the virtual oscilloscope. Panel (b) represents the phase portrait obtained by supplying to the horizontal and vertical inputs the voltages on the capacitors $\mathrm{C} 1$ for the blocks separated by half the length of the chain, i.e., a quarter of the wavelength of the main mode. Comparison with the portraits of the attractor obtained from the results of the numerical integration of the equations shown in Figs. 5a, 5b demonstrates excellent agreement.
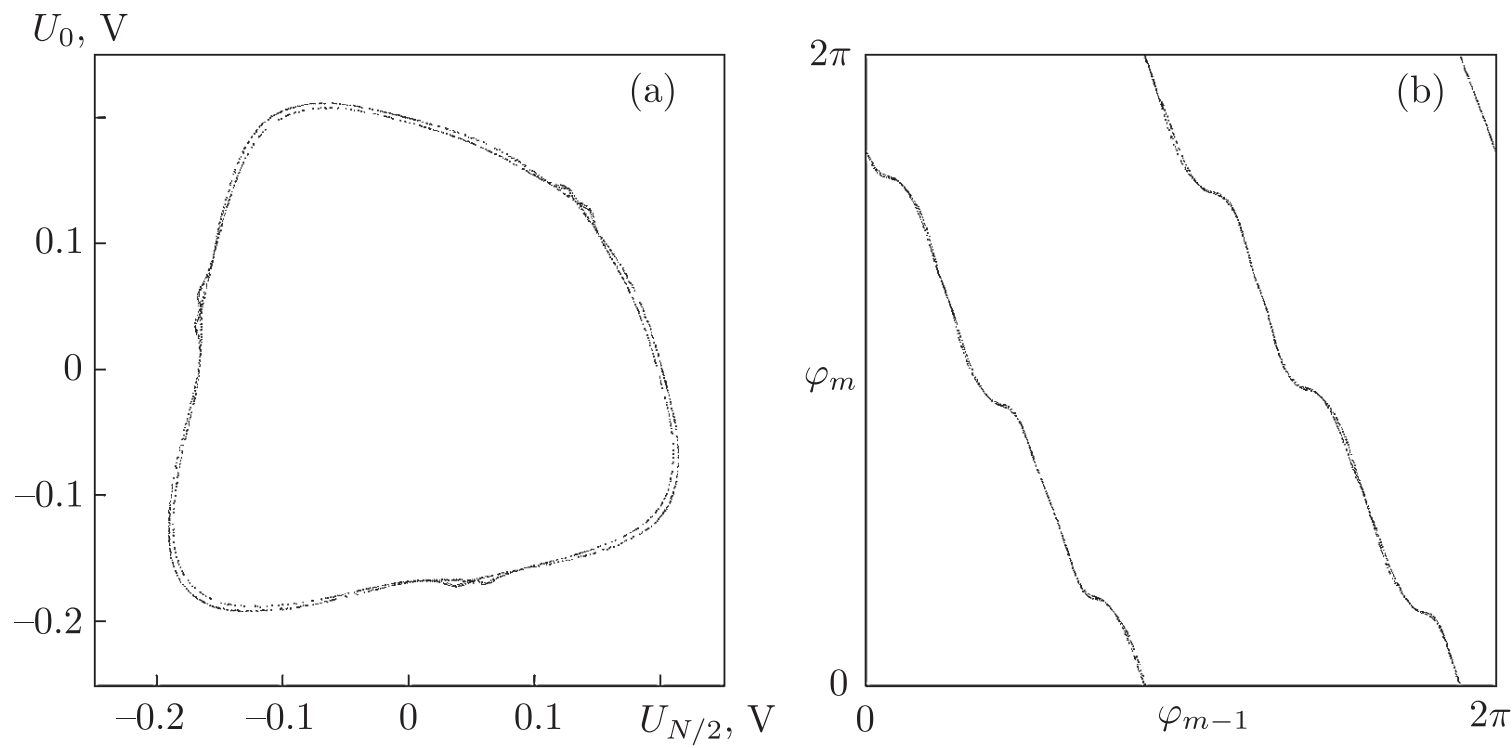

Fig. 11. Set of points $\left(U_{0}, U_{N / 2}\right)$, corresponding to moments of reaching the maximum values $\sqrt{U_{0}^{2}+U_{3}^{2}}$ visualizing the Smale-Williams solenoid in the projection on the plane (a), and the diagram of transformation of the spatial phase $\varphi_{m}=\arg \left(U_{0}+i U_{N / 2}\right)$ (b) from results of processing the simulation data in the Multisim environment.

To visualize the attractor in the Poincaré section and a diagram that would illustrate the transformation of the angular variable characteristic for the Smale-Williams attractor, we have to turn to recording the simulation data in the Multisim environment to a file with their subsequent processing by a specially composed program. To do this, we use the same oscilloscope connection as in the case of visualization of the attractor in Fig. 10b, and the Grapher tool, which provides the ability to record simulation data in the Multisim environment. The sampling of the voltage values $U_{0}$ and $U_{3}$ is performed with a time step of three orders less than the characteristic period of the succesive stages of activity. Then the file is processed by the program, which, reading the recorded data step by step, calculates the value characterizing the instantaneous amplitude of the basic spatial mode. At the moments when this value reaches a local maximum of a level above $0.01 \mathrm{~V}$, the dots are plotted in coordinates $U_{0}$ and $U_{3}$, which form the portrait of the attractor in the Poincaré section as shown in Fig. 11a. At the same moments, the values of the angular variable $\varphi_{m}=\arg \left(U_{0}\left(t_{m}\right)+i U_{0}\left(t_{m}\right)\right)$ are calculated and plotted in coordinates $\left(\varphi_{m-1}, \varphi_{m}\right)$ (Fig. 11b). As seen, the portrait of the attractor in the Poincaré section corresponds to the projection of the Smale-Williams solenoid with its characteristic transverse filament structure. The diagram for the angular variable corresponds to a double-expanding circle map, which has to be regarded as the main confirmation of the hyperbolic nature of the 
attractor observed in the circuit simulation in the proposed CNN. Note that visually the graphs in Fig. 11 and the diagrams obtained from the results of the numerical solution of the equations in Figs. 5c and 3 are in very good agreement.

\section{Conclusion}

This article discusses a specific implementation of a novel electronic system that demonstrates the generation of chaos, which has the property of roughness or structural stability due to the presence of a uniformly hyperbolic attractor. This is another example of a Smale-Williams type attractor in an autonomous electronic circuit, in addition to the one described earlier in [47].

The study indicates a new interesting direction in the use of cellular neural networks, namely, to build robust chaos generators. Although the case of $N=6$ was considered here as a basic example, it is rather obvious from the content of Section 2 that the same type of rough chaos can be obtained in CNN composed of a larger number of cells as well.

The proposed circuit is the first example where the role of the angular coordinate for the Smale-Williams attractor is played by the spatial phase of the sequence of patterns formed in a spatially extended system. It thus implements the principle of operation, similar to the mechanism described in [49-51]. (In systems proposed earlier, in most cases, the temporal phase of oscillatory processes was treated as an angular variable $[43,44]$.)

Very satisfactory spectral properties of the chaos generator should be noted; it is characterized by a smooth distribution of the spectral power density in frequency not containing visible peaks and dips.

In the future, it would be interesting to implement the described scheme in a real experiment and to consider the possibility of its implementation in the form of a microchip, as well as a passage to the range of high and ultrahigh frequencies.

The system considered supplements the collection of feasible systems with hyperbolic attractors and thus contributes to fleshing out the hyperbolic theory, which constitutes an important and deep branch of the modern mathematical theory of dynamical systems.

\section{References}

[1] Chua, L. O. and Yang, L., Cellular Neural Networks: Theory, IEEE Trans. Circuits Syst., 1988, vol. 35, no. 10, pp. 1257-1272.

[2] Chua, L. O. and Roska, T., The CNN Paradigm, IEEE Trans. Circuits Syst. I, 1993, vol.40, no. 3, pp. $147-156$.

[3] Cimagalli, V., Balsi, M., and Caianiello, E., Cellular Neural Networks: A Review, in Neural Nets WIRN Vietri'93: Proc. of 6th Italian Workshop (Salerno, 1993), E. R. Caianiello (Ed.), World Sci., 1993, pp. 55-84.

[4] Chua, L. O. and Yang, L., Cellular Neural Networks: Applications, IEEE Trans. Circuits Syst., 1988, vol. 35, no. 10, pp. 1273-1290.

[5] Chua, L. O., Hasler, M., Moschytz, G. S., and Neirynck, J., Autonomous Cellular Neural Networks: A Unified Paradigm for Pattern Formation and Active Wave Propagation, IEEE Trans. Circuits Syst. I, 1995, vol. 42, no. 10, pp. 559-577.

[6] Hunt, K. J., Sbarbaro, D., Żbikowski, R., and Gawthrop, P. J., Neural Networks for Control Systems: A Survey, Automatica, 1992, vol. 28, no.6, pp. 1083-1112.

[7] Chua, L. O. and Roska, T., Cellular Neural Networks and Visual Computing: Foundations and Applications, Cambridge: Cambridge Univ. Press, 2002. 
[8] Shi, B. and Luo, T., Spatial Pattern Formation via Reaction-Diffusion Dynamics in $32 \times 32 \times 4$ CNN Chip, IEEE Trans. Circuits Syst. I, 2004, vol.51, no. 5, pp. 939-947.

[9] Gollas, F. and Tetzlaff, R., Modeling Complex Systems by Reaction-Diffusion Cellular Nonlinear Networks with Polynomial Weight-Functions, in 9th Internat. Workshop on Cellular Neural Networks and Their Applications (Taiwan, 2005), pp. 227-231.

[10] Pivka, L., Autowaves and Spatio-Temporal Chaos in CNNs: 1. A Tutorial, IEEE Trans. Circuits Syst. I, 1995, vol. 42, no. 10, pp. 638-649.

[11] Chaotic Electronics in Telecommunications, M.Kennedy, G. Setti, R. Rovatti (Eds.), Boca Raton, Fla.: CRC, 2000.

[12] Cuomo, K. M. and Oppenheim, A. V., Circuit Implementation of Synchronized Chaos with Applications to Communications, Phys. Rev. Lett., 1993, vol.71, no. 1, pp. 65-68.

[13] Dmitriev, A. S., Panas, A. I., and Starkov, S. O., Experiments on Speech and Music Signals Transmission Using Chaos, Internat. J. Bifur. Chaos Appl. Sci. Engrg., 1995, vol. 5, no. 4, pp. 1249-1254.

[14] Bollt, E. M., Review of Chaos Communication by Feedback Control of Symbolic Dynamics, Internat. J. Bifur. Chaos Appl. Sci. Engrg., 2003, vol.13, no. 2, pp. 269-285.

[15] Baptista, M. S., Cryptography with Chaos, Phys. Lett. A, 1998, vol. 240, nos. 1-2, pp. 50-54.

[16] Kocarev, L., Chaos-Based Cryptography: A Brief Overview, IEEE Circuits Syst. Mag., 2001, vol.1, no. 3, pp. 6-21.

[17] Dachselt, F. and Schwarz, W., Chaos and Cryptography, IEEE Trans. Circuits Syst. I, 2001, vol. 48, no. 12 , pp. $1498-1509$.

[18] Stojanovski, T. and Kocarev, L., Chaos-Based Random Number Generators: Part 1. Analysis [Cryptography], IEEE Trans. Circuits Syst. I, 2001, vol.48, no. 3, pp. 281-288.

[19] Stojanovski, T., Pihl, J., and Kocarev, L., Chaos-Based Random Number Generators: Part 2. Practical Realization, IEEE Trans. Circuits Syst. I, 2001, vol.48, no. 3, pp. 382-385.

[20] Bakiri, M., Guyeux, C., Couchot, J.F., and Oudjida, A. K., Survey on Hardware Implementation of Random Number Generators on FPGA: Theory and Experimental Analyses, Comput. Sci. Rev., 2018, vol. 27, pp. 135-153.

[21] Verschaffelt, G., Khoder, M., and Van der Sande, G., Random Number Generator Based on an Integrated Laser with On-Chip Optical Feedback, Chaos, 2017, vol. 27, no. 11, 114310, 7 pp.

[22] Harman, S. A., Fenwick, A. J., and Williams, C., Chaotic Signals in Radar?, in Proc. of the 3rd European Radar Conference IEEE (Manchester, September 2006), pp. 49-52.

[23] Liu, Z., Zhu, X., Hu, W., and Jiang, F., Principles of Chaotic Signal Radar, Internat. J. Bifur. Chaos Appl. Sci. Engrg., 2007, vol.17, no. 5, pp. 1735-1739.

[24] Willsey, M.S., Cuomo, K. M., and Oppenheim, A. V., Selecting the Lorenz Parameters for Wideband Radar Waveform Generation, Internat. J. Bifur. Chaos Appl. Sci. Engrg., 2011, vol. 21, no.9, pp. 2539-2545.

[25] Banerjee, S., Yorke, J. A., and Grebogi, C., Robust Chaos, Phys. Rev. Lett., 1998, vol. 80, no. 14, pp. 3049-3052.

[26] Potapov, A. and Ali, M. K., Robust Chaos in Neural Networks, Phys. Lett. A, 2000, vol. 277, no. 6, pp. 310-322.

[27] Elhadj, Z. and Sprott, J. C., On the Robustness of Chaos in Dynamical Systems: Theories and Applications, Front. Phys. China, 2008, vol.3, no. 2, pp. 195-204.

[28] Elhadj, Z. and Sprott, J. C., Robust Chaos and Its Applications, World Sci. Ser. Nonlinear Sci. Ser. A Monogr. Treatises, vol. 79, Hackensack, N.J.: World Sci., 2011.

[29] Gusso, A., Dantas, W. G., and Ujevic, S., Prediction of Robust Chaos in Micro and Nanoresonators under Two-Frequency Excitation, Chaos, 2019, vol. 29, no. 3, Art. 033112.

[30] Shilnikov, L., Mathematical Problems of Nonlinear Dynamics: A Tutorial, Internat. J. Bifur. Chaos Appl. Sci. Engrg., 1997, vol. 7, no. 9, pp. 1953-2001. 
[31] Botella-Soler, V., Castelo, J. M., Oteo, J. A., and Ros, J., Bifurcations in the Lozi Map, J. Phys. A, 2011, vol. 44, no. 30, 305101, 14 pp.

[32] Elhadj, Z., Lozi Mappings: Theory and Applications, Boca Raton, Fla.: CRC, 2013.

[33] Belykh, V. N. and Belykh, I., Belykh Map, Scholarpedia, 2011, vol.6, no. 10, p. 5545.

[34] Kuznetsov, S.P., Belykh Attractor in Zaslavsky Map and Its Transformation under Smoothing, Izv. Vyssh. Uchebn. Zaved. Prikl. Nelin. Dinam., 2018, vol. 26, no. 1, pp. 64-79 (Russian); see also (in English): arXiv:1710.07828 (2017).

[35] Anosov, D. V., Dynamical Systems in the 1960s: The Hyperbolic Revolution, in Mathematical Events of the Twentieth Century, A. A. Bolibruch, Yu. S. Osipov, Ya. G. Sinai (Eds.), Berlin: Springer, 2006, pp. 1-17.

[36] Smale, S., Differentiable Dynamical Systems, Bull. Amer. Math. Soc., 1967, vol.73, no. 6, pp. 747817.

[37] Dynamical Systems 9: Dynamical Systems with Hyperbolic Behaviour, D. V. Anosov (Ed.), Encyclopaedia Math. Sci., vol. 66, Berlin: Springer, 1995.

[38] Katok, A. and Hasselblatt, B., Introduction to the Modern Theory of Dynamical Systems, Encyclopedia Math. Appl., vol. 54, Cambridge: Cambridge Univ. Press, 1995.

[39] Pugh, C. and Peixoto, M. M., Structural Stability, Scholarpedia, 2008, vol.3, no. 9, p. 4008.

[40] Kuznetsov, S. P., Example of a Physical System with a Hyperbolic Attractor of the Smale-Williams Type, Phys. Rev. Lett., 2005, vol. 95, no. 14, 144101, 4 pp.

[41] Kuznetsov, S. P. and Pikovsky, A., Autonomous Coupled Oscillators with Hyperbolic Strange Attractors, Phys. D, 2007, vol. 232, no. 2, pp. 87-102.

[42] Wilczak, D., Uniformly Hyperbolic Attractor of the Smale-Williams Type for a Poincaré Map in the Kuznetsov System: With Online Multimedia Enhancements, SIAM J. Appl. Dyn. Syst., 2010, vol. 9, no. 4, pp. 1263-1283.

[43] Kuznetsov, S.P., Dynamical Chaos and Uniformly Hyperbolic Attractors: From Mathematics to Physics, Phys. Uspekhi, 2011, vol.54, no.2, pp.119-144; see also: Uspekhi Fiz. Nauk, 2011, vol. 181, no. 2, pp. 121-149.

[44] Kuznetsov, S. P., Hyperbolic Chaos: A Physicist's View, Berlin: Springer, 2012.

[45] Kuznetsov, S. P. and Seleznev, E.P., Strange Attractor of Smale-Williams Type in the Chaotic Dynamics of a Physical System, J. Exp. Theor. Phys., 2006, vol.102, no. 2, pp. 355-364; see also: Zh. Èksper. Teoret. Fiz., 2006, vol. 129, no. 2, pp. 400-412.

[46] Kuznetsov, S. P. and Ponomarenko, V. I., Realization of a Strange Attractor of the Smale-Williams Type in a Radiotechnical Delay-Feedback Oscillator, Tech. Phys. Lett., 2008, vol. 34, no. 9, pp. 771773; see also: Pisma Zh. Tekh. Fiz., 2008, vol.34, no. 18, pp.1-8.

[47] Kuznetsov, S. P., Ponomarenko, V.I., and Seleznev, E. P., Autonomous System Generating Hyperbolic Chaos: Circuit Simulation and Experiment, Izv. Vyssh. Uchebn. Zaved. Prikl. Nelin. Dinam., 2013, vol. 21, no. 5, pp. 17-30 (Russian).

[48] Isaeva, O. B., Kuznetsov, S. P., Sataev, I. R., Savin, D. V., and Seleznev, E. P., Hyperbolic Chaos and Other Phenomena of Complex Dynamics Depending on Parameters in a Nonautonomous System of Two Alternately Activated Oscillators, Internat. J. Bifur. Chaos Appl. Sci. Engrg., 2015, vol. 25, no. 12, 1530033, 15 pp.

[49] Kuptsov, P. V., Kuznetsov, S. P., and Pikovsky, A. Hyperbolic Chaos of Turing Patterns, Phys. Rev. Lett., 2012, vol. 108, no. 19, 194101, 4 pp.

[50] Isaeva, O. B., Kuznetsov, A.S., and Kuznetsov, S. P., Hyperbolic Chaos of Standing Wave Patterns Generated Parametrically by a Modulated Pump Source, Phys. Rev. E, 2013, vol. 87, no.4, 040901(R), 4 pp.

[51] Kruglov, V.P., Kuznetsov, S. P., and Pikovsky, A., Attractor of Smale-Williams Type in an Autonomous Distributed System, Regul. Chaotic Dyn., 2014, vol.19, no. 4, pp. 483-494. 
[52] Benettin, G., Galgani, L., Giorgilli, A., and Strelcyn, J.-M., Lyapunov Characteristic Exponents for Smooth Dynamical Systems and for Hamiltonian Systems: A Method for Computing All of Them: P. 1: Theory, Meccanica, 1980, vol. 15, no. 1, pp. 9-20.

[53] Shimada, I. and Nagashima, T., A Numerical Approach to Ergodic Problem of Dissipative Dynamical Systems, Progr. Theoret. Phys., 1979, vol.61, no. 6, pp. 1605-1616.

[54] Pikovsky, A. and Politi, A., Lyapunov Exponents: A Tool to Explore Complex Dynamics, Cambridge: Cambridge Univ. Press, 2016.

[55] Kaplan, J.L. and Yorke, J.A., Chaotic Behavior of Multidimensional Difference Equations, in Functional Differential Equations and Approximation of Fixed Points, H.-O. Peitgen, H.-O. Walther (Eds.), Lecture Notes in Math., vol. 730, Berlin: Springer, 1979, pp. 204-227.

[56] Farmer, J. D., Ott, E., and Yorke, J. A., The Dimension of Chaotic Attractors, Phys. D, 1983, vol. 7, nos. $1-3$, pp. $153-180$. 